\title{
Multiple Feature Extraction Techniques in Image Stitching
}

\author{
Taherim S. Shaikh \\ Student, M.E. (Computer Engineering) \\ Thadomal Shahani Engineering College \\ Mumbai, India
}

\author{
Archana B. Patankar, PhD \\ Associate Professor, Computer Department \\ Thadomal Shahani Engineering College \\ Mumbai, India
}

\begin{abstract}
Image stitching is the process of combining two or more images with overlapping area to produce a panorama of input images. In order to improve performance of creation of panorama, approach that combine different keypoint extraction methods can be used.

This paper proposes a methodology for image stitching process that combines various feature detection and extraction algorithms. First, image stitching will be done based on feature keypoint matches. Final image with seam will be blended with image blending technique.

The paper summarizes 3 robust feature detection and extraction algorithms namely SURF, SIFT and MSER. Combined set of keypoints will be used for image transformation. According to proposed system, multiple feature extraction techniques can be used for image stitching which can build seamless panorama image.
\end{abstract}

\section{General Terms}

Image Processing, Image, Image stitching Algorithm, Feature extraction algorithm, Image blending

\section{Keywords}

Keypoints, Detectors, Descriptors, SURF, SIFT, MSER, RANSAC, FLANN

\section{INTRODUCTION}

During recent years, keypoint extraction and matching is one of the most important research areas in the field of image processing. It is always better to pick 100 important points than picking 100 random points in an image. Few parts of an image may have some extra information than others (especially at edges and corners), and these are the ones you should use for better image matching.

The detection, description and matching of point features plays an important role in most of the image processing algorithms[1][2].Over the last years several new fast detectors(FAST[12],SURF[2],SIFT[1]STAR[12])and various descriptors(SURF[6],BRIEF[8],ORB[9], FREAK [10]) have been proposed and successfully applied to multiple tasks.

This paper proposes an approach which uses combination of three robust feature detection algorithms namely SIFT (Scale Invariant Feature Transform), SURF (Speeded Up Robust Feature) and MSER (Maximally Stable Extremal Region) for constructing panorama of input images.

This papers present the comparison and evaluation of the SURF, SIFT and MSER feature detection algorithms. Results of individual feature detection algorithms are provided in section 4. Depending on advantages of various feature detection and extraction techniques, combinations of various algorithms are used to improve the quality of final panorama image.

The remaining part of paper is structured as follows: Section 2, states background research and literature review. Section 3 explains the proposed method. Section 4, shows experiment and results. Section 5 gives conclusion remark and future work.

\section{BACKGROUND AND LITERATURE REVIEW}

Nowadays, image stitching technology is one of the most emerging and trending topic in computer graphics. In recent years, the image mosaic technology has become a popular research topic in image processing. It can stitch the images which have overlapped area to a higher resolution or wideangle image. Traditional applications include construction of aerial and satellite photographs. Nowadays, the image mosaic technology is widely applied in remote sensing image processing, medical image analysis, the realization of virtual reality, the processing of satellite images, the military, etc. The image mosaic method based on SIFT [4] (Scale Invariant Feature Detection) feature matching has strong robustness, even if the image at different scales can be stitched. However, the computing of SIFT operator takes so long which leads to low efficiency of mosaic. In 2006, Bay, etc. proposed (Speeded Up Robust Features) SURF [3] operator, similar to SIFT, it also has few good characteristics like scale invariance, better robustness and good variation between feature points; Compared with the SIFT operator, it have greatly improved in processing speed [3]. In case of blending we face the problem of optical seam, and to provide seamless results we can enhance blending technique.

\subsection{SURF and SIFT}

So far, SIFT and SURF are the two most popular and accurate descriptors, which shares a lot of similarities. First, the input normalized region is required to be with the resolution $16 \times 16$. Then the $16 \times 16$ region is subdivided into 16 blocks of a $4 \times 4$ resolution, in which, 16 blocks are used to encode the geometric information, while the $4 \times 4$ subregion is used to normalized to the localization error and noise. Moreover, both algorithms use the statistics of the gradients to gain photometric invariance. However, the main difference of these two descriptors lies in the part about how to encode the information in each $4 \times 4$ subregion. SIFT make a 8 bins histogram of the gradients inside this region by every $45^{\circ}$, and let each gradient vote to two neighboring bins it belongs to, to make it more robust to 36 localization error. On the other hand, SURF calculates the following statistics of gradient $\mathrm{Pdx}, \mathrm{Pdy}, \mathrm{P}|\mathrm{dx}|$, and P $|\mathrm{dy}|$. 
SURF and SIFT detectors can detect features like corner and blob but can't detect keypoints around region. Both are invariant to rotation and scale but not affine.

\subsection{MSER}

MSER is the method for blob detection in images which denotes a set of different regions which are defined by an extremal property of its intensity function in the region and on its outer boundary. Previously it was used to find correspondences between image elements with different viewpoints from two images. MSER [7] can detect features around region of an object but can't detect corner and blob features. MSER is most efficient algorithm since it is invariant to rotation, scale as well as affine transformation.

In comparison with other approaches, using these 3 together can overcome all the limitations and can give perfect results.

\subsection{Advantages of SURF, SIFT and MSER}

The main advantages of the SIFT and SURF descriptors are that they are quite robust to noise and error detection, and keep invariant to photometric changes like scale, rotation, etc. Due to the zero mean of the white noise, the histogram in SIFT and the summation in SURF, can suppress noise to a low level. The statistics of the gradient over an angle of $45^{\circ}$ makes SIFT robust to localization error, while the summation of gradient over the $4 \times 4$ block of SURF makes it robust to localization error too. Moreover, because both of SIFT and SURF use the statistics of gradient, which is invariant to photometric changes, they both are robust to illumination changes. However, there are two significant problems about these two descriptors.

The first problem about these two descriptor is that the feature vector is too large to give a compact representation of the point. For the original region without any operation of descriptor, the length of feature vector is $16 \times 16=256$, while for SIFT is $16 \times 8=128$ and $16 \times 4=64$ for SURF. Usually, for one image, there would be hundreds or thousands of interest points detected, thus, the length of the feature vector would play a very important role in the computational complexity of the matching stage. For this kind of consideration, SIFT only has a compression factor of 2, and SURF gains 4 , while actually the subregion usually contains quite few information.

The second problem is that, the required input region need to have a size of $16 \times 16$. Thus, if the size of region is much smaller than this, for example, a $4 \times 4$ region, then the descriptor would still build up a feature vector of length of 128 or 64. In some sense, the descriptor increase information, which should never happen. Thus, there is no wonder why in Image Retrieval, there is some low resolution images can matches to whatever images.

\section{Table 1: Feature Detectors Overview}

\begin{tabular}{|c|ccc|ccc|cccc|}
\hline $\begin{array}{c}\text { Feature } \\
\text { Detector }\end{array}$ & Comer Blob Region & $\begin{array}{l}\text { Rotation } \\
\text { Invariant }\end{array}$ & $\begin{array}{c}\text { Scale } \\
\text { Invariant }\end{array}$ & $\begin{array}{c}\text { Affine } \\
\text { Invariant }\end{array}$ & $\begin{array}{c}\text { Repeat } \\
\text { ability }\end{array}$ & $\begin{array}{c}\text { Location } \\
\text { Accuracy }\end{array}$ & $\begin{array}{c}\text { Robus } \\
\text { thess }\end{array}$ & $\begin{array}{c}\text { effici } \\
\text { ency }\end{array}$ \\
\hline Harris & + & & & + & & & +++ & +++ & +++ & ++ \\
\hline SURF & + & + & & + & + & & ++ & ++ & ++ & +++ \\
\hline Hessian & & + & & + & & & ++ & ++ & ++ & + \\
\hline MSER & & & + & + & + & + & +++ & +++ & ++ & +++ \\
\hline DoG & + & + & & + & + & & ++ & ++ & ++ & ++ \\
\hline SIFT & + & + & & + & + & & ++ & ++ & ++ & +++ \\
\hline
\end{tabular}

Table 1 gives overview of various feature detector algorithms. All has some advantages and few disadvantages. We propose a method which overcomes these limitations. Using SURF, SIFT and MSER together we can have better set of keypoints which will result in perfect panorama result.

\section{PROPOSED METHOD}

The image stitching methodology is divided into 4 phases. In this first user will select input overlapping images which are to be stitched. Image registration and merging takes place to get final results. Image registration consists of feature matching which is done by creating feature detector and feature descriptor technique. Feature keypoints will include combination of keypoint dataset created by SURF [1], SIFT [2], MSER [7] feature detector algorithms.

The technique proposed below, a panorama image stitching system focuses on image registration part. In case of image registration, we match the features of images using some feature detection technique and depending on this output will be generated. The output of image registration will give you higher quality of image stitching i.e. your final image. We get keypoint detectors and keypoint descriptors from feature matching. The better the feature matching is, more matches will found. Based on large number of matches we can transform or overlay 2 images in efficient manner. Entire stitching is bases on feature extraction and matching. So, the proposed system focuses on feature extraction part of image stitching technique. Proposed method has 4 basic steps.

$$
\begin{array}{ll}
\text { - } & \text { Feature Extraction } \\
\text { - } & \text { Feature matching } \\
\text { - } & \text { Image transformation } \\
\text { - } & \text { Image blending }
\end{array}
$$

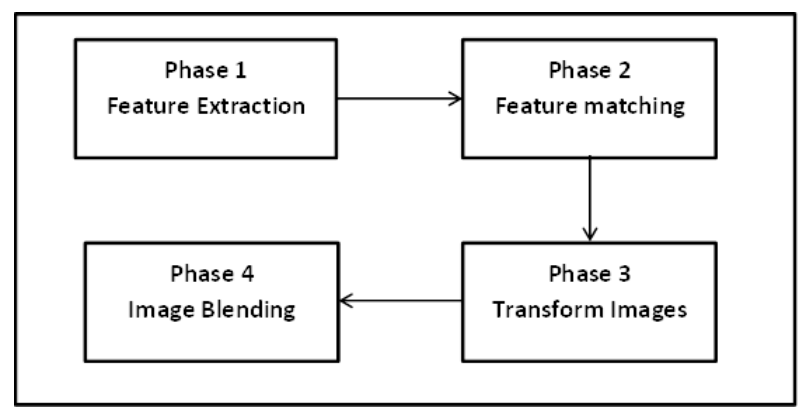

Fig. 1: Phases of image stitching method 


\subsection{Feature Extraction}

Depending on the image content, some of these image structures are more common than others, thus the number of features found with a given detector may vary for different image categories Detect keypoints in both images using SIFT, SURF and MSER[5] feature extraction technique remove duplicates and compute the feature keypoints. Compute feature descriptor of matching keypoints. Computing local features consists of detecting salient locations such as corner, blob and region detectors. Get the keypoints. SIFT SURF and MSER feature keypoints will be computed first. Then corresponding feature descriptors will be calculated.

\subsection{Feature Matching}

FLANN (fast library for approximate nearest neighbor) will be used for feature matching. Get best feature keypoint matches using FLANN [10]. It is the simplest method of classification available for supervised learning. The main goal is to search for closest match of the test data in space of feature. Match descriptor vectors using FLANN matcher [9]. Calculate max and min distances between keypoints. Use only good matches whose distance is less than $3 *$ min_dist. Get the keypoints from good matches

\subsection{Image transformation}

Find Homography matrix to warp images. To find the better transform between two sets of points, findHomography is used. The base for findHomography is GetPerspectiveTransform, and it is useful in many situations where you only have 4 correct points. The findHomography is used with sets of points detected automatically. GetPerspectiveTransform is meant to work on 3 or 4 points (respectively) that are known to be correct correspondences. Image will be translated to the same plane.

\subsection{Image Blending}

After images are stitched, there are chances it may have seam so here main goal is to produce an image where no transition is present between the original source images. The blending technique will be used for removing seam.

Multi-band blending (also known as pyramid blending): It is widely used for image stitching without ghosting and blurring effects [11]. It will give much better results than any other blending technique. Multiband blending scheme gives smooth transitions between images despite illumination differences. We will be using multi band technique to remove seam in final stitched image to get perfect seamless panorama.

Fig 2 shows the implementation details of proposed method. First user will select input images which have to be stitched. If there are any noises in images that will be removed using fast non local based denoising algorithm. Feature keypoints will be extracted from input images using SURF, SIFT and MSER feature detector algorithms. Combined set of keypoints will be formed. Feature descriptors will be calculated and duplicate keypoints will be removed. Good matches will be found from input images using FLANN matcher [9]. Panorama with seam will be created after image transformation will be done. Lastly Multiband image blending technique will be used for removing seam from panorama.

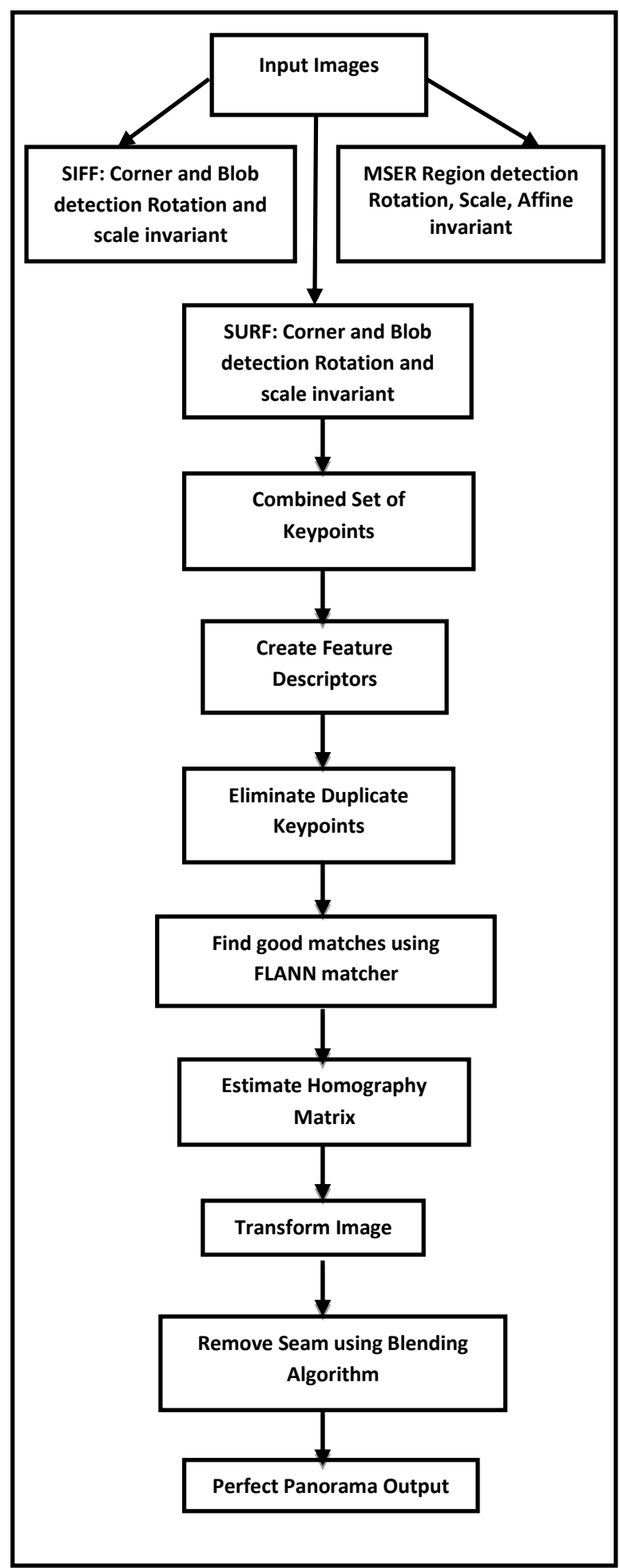

Fig. 2: Proposed framework for image stitching 


\section{EXPERIMENTS AND RESULTS}

Experiment consists of 2 parts. First combined set of keypoints will be produced using SURF, SIFT and MSER and then image stitching will be done followed by image blending technique.

Table 2: The number of Matched points of SURF and SIFT in change of scale

\begin{tabular}{|c|c|c|}
\hline $\begin{array}{c}\text { Object dataset } \\
\text { Image number }\end{array}$ & $\begin{array}{c}\text { SIFT } \\
\text { (repeatability) }\end{array}$ & $\begin{array}{c}\text { SURF } \\
\text { (repeatability) }\end{array}$ \\
\hline $11-12$ & $43 \%$ & $70 \%$ \\
\hline $11-13$ & $32 \%$ & $49 \%$ \\
\hline $11-14$ & $18 \%$ & $25 \%$ \\
\hline $11-15$ & $8 \%$ & $6 \%$ \\
\hline $11-16$ & $2 \%$ & $5 \%$ \\
\hline Average & $21 \%$ & $31 \%$ \\
\hline
\end{tabular}

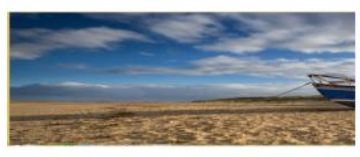

input image 1

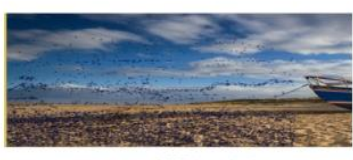

SURF keypoint detector

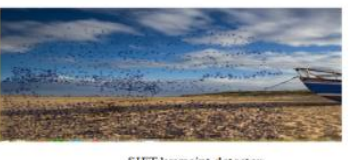

SIFT keypoint detector

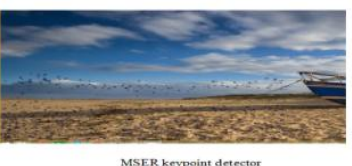

Fig. 3: Data set 1

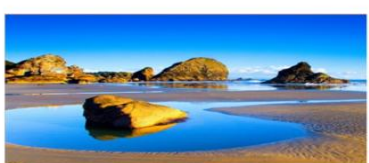

input image 2

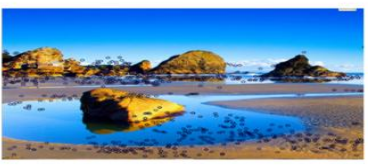

SURF keypoint detector

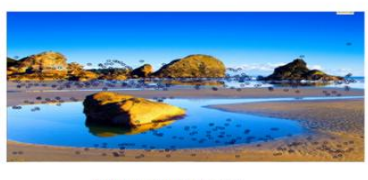

SIFT keypoint detector

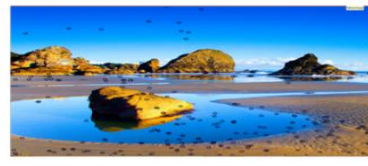

MSER keypoint detectot
Fig. 4: Data set 2

Fig 3 and 4 shows keypoint detection for input images using SIFT, SURF and MSER algorithm. Input images shown in the fir 3 and 4 will undergo in SURF, SIFT and MSER feature detection techniques.
Table 3: Repeatability of SURF and SIFT in change of illumination

\begin{tabular}{|c|c|c|}
\hline $\begin{array}{c}\text { Object dataset } \\
\text { Image number }\end{array}$ & SIFT & SURF \\
\hline $1-2$ & 205 & 246 \\
\hline $3-4$ & 156 & 146 \\
\hline $5-6$ & 106 & 87 \\
\hline $7-8$ & 75 & 18 \\
\hline $9-10$ & 0 & 2 \\
\hline
\end{tabular}

Table 2 shows that SURF and SIFT are equally robust in scale change and Table 3 shows that SURF is more robust than SIFT in case of illumination change. SURF is not better than SIFT in rotation, but SURF is as robust as SIFT in other performance. In panorama image stitching, there is no large rapid rotation generally, so this paper chooses SURF, SIFT and MSER to be the feature detection methods weighted on its time cost and its good performance in case of illumination and scale changes.

\section{CONCLUSION AND FUTURE WORK}

Panorama image stitching technique is one of the trending and important topics in computer vision and graphics. Image stitching algorithm has wide range of application in various domains such as art, theatre, photography, etc. The proposed algorithm can give best results and perfect panorama.

The proposed method is based on image stitching using multiple feature detection algorithms like SURF, SIFT and MSER. Results of individual algorithms are showed. This system can give better performance than existing systems. But the present system shows some defects when there is some noise present in images. So as a future work, we plan to do more research in the area through removing the noise before stitching processing. More than 2 input images i.e. 8 can be stitched together using this technique. Performance Evaluation of this technique is yet to be done.

\section{REFERENCES}

[1] Luo Juan1, Oubong Gwun, "SURF applied in Panorama Image Stitching", IEEE Computer Graphics Lab, Computer Science \& Computer Engineering,Chonbuk National University, Jeonju 561-756, South Korea,2010.

[2] Bay H, Tuytelaars T, van Gool L J.SURF: Speeded Up Robust Features[C]//European Conference on Computer Vision, 2006, I: 404-417.

[3] Aathreya S. Bhat, Amith V. Shivaprakash, Namrata S. Prasad, Chaitra Nagaraj "Template Matching Technique for Panoramic Image Stitching",IEEE Transactions On Image Processing, 978-0-7695-5101-2/13 (C) 2013 IEEE. 
[4] Meng You, Jong Seok Lim, Wook Hyun Kim, "Panoramic image stitching using SURF", Yeungam univercity.

J. Matas, O. Chum, M. Urban, T. Pajdla:"Robust Wide Baseline stereo from maximally stable extreamal regions", British machine vision conference, 384-393,2002.

[5] Maja Rudinac, Boris Lenseigne, Pieter Jonker, "Keypoint extraction and selection for object recognition",MVA2009 IAPR conference on Machine Vision Applications, May 20-22,2009, Yokohama,JAPAN.

[6] J.M. Morel and G. Yu. ASIFT: A new framework for fully affine invariant image comparison. SIAM Journal on Imaging Sciences, 2(2):438-469, 2009.

[7] Donoser, M.; Bischof, H. "Efficient Maximally Stable Extremal Region (MSER) Tracking". IEEE Computer Society Conference on Computer Vision and Pattern Recognition, June 2006, pp.553-56.
[8] Luo Juan and Oubong Gwun, - A Comparison of SIFT, PCA-SIFT and SURF\| International Journal of Image Processing (IJIP), Volume(3): Issue (4) 2009.

[9] Marius Muja, David G. Lowe," FAST APPROXIMATE NEAREST NEIGHBORS WITH AUTOMATIC ALGORITHM CONFIGURATION", Computer Science Department, University of British Columbia, Vancouver, B.C., Canada.

[10] Cedric All ' ene, Jean-Philippe Pons and Renaud Keriven, "Seamless Image-Based Texture Atlases using Multi-band Blending", CERTIS, Ecole des ponts, Paris-Est, France.

[11] Adam Schimidit, Marek Kraft, Michat Fularz, Zuzanna Domagala,"THE COMPARISON OF POINT FEATURE DETETORS AND DESCRIPTORS AND DESCRIPTORS IN THE CONTEXT OF ROBOT NAVIGATION",Workshop on Perception for Mobile Robots Autonomy 2012. 